\title{
A COMMUTATIVITY-OR-FINITENESS CONDITION FOR RINGS
}

\section{ABRAHAM A. KLEIN and HOWARD E. BELL}

Received 20 May 2004

\begin{abstract}
We show that a ring with only finitely many noncentral subrings must be either commutative or finite.
\end{abstract}

2000 Mathematics Subject Classification: 16U80, 16 P99.

1. Preliminaries. In [1] the following theorem was proved.

THEOREM 1.1. If $R$ is a periodic ring having only finitely many noncentral subrings of zero divisors, then $R$ is either finite or commutative.

In view of this result, it was conjectured that any ring with only finitely many noncentral subrings is either finite or commutative. It is our principal goal to prove this conjecture; and in the process we provide a new proof of Theorem 1.1.

For any ring $R$, the symbols $N, D, Z$, and $\wp(R)$ will denote, respectively, the set of nilpotent elements, the set of zero divisors, the center, and the prime radical; and $R$ will be called reduced if $N=\{0\}$.

We will require the following three lemmas.

LEMMA 1.2 [5, Corollary 5]. If $R$ is any ring in which $N$ is finite, then $R / \wp(R)=B \oplus C$, where $B$ is reduced and $C$ is a direct sum of finitely many total matrix rings over finite fields.

LEMMA 1.3. If $R$ is any ring in which $\wp(R) \subseteq Z$, then zero divisors in $R / \wp(R)$ may be lifted to zero divisors in $R$.

Proof. We show that if $\bar{x}=x+\wp(R)$ is a zero divisor in $\bar{R}=R / \wp(R)$, then $x$ is a zero divisor in $R$. Clearly, this is the case for $\bar{x}=\overline{0}$, so we assume that $\bar{x} \neq \overline{0}$ and that $\bar{y} \in \bar{R} \backslash\{\overline{0}\}$ is such that $\bar{x} \bar{y}=\overline{0}$. Then, $x y \in \wp(R)$, and there exists $n$ such that $(x y)^{n}=0$. Moreover, since $x y r \in \wp(R)$ for all $r \in R$, we have $x y \in Z$ and $x y r \in Z$ for all $r \in R$.

Since $y \notin \wp(R), y$ is not strongly nilpotent, therefore there exist $r_{1}, r_{2}, \ldots, r_{n-1} \in R$ such that $y r_{1} y r_{2} \cdots y r_{n-1} y \neq 0$. Since $x y$ and all $x y r_{i}$ are central, we get $x^{n} y r_{1} y r_{2}$ $\cdots y r_{n-1} y=x y r_{1} x^{n-1} y r_{2} \cdots y=x y r_{1} x y r_{2} x^{n-2} \cdots y=x y r_{1} x y r_{2} \cdots x y r_{n-1} x y$ $=(x y)^{n} r_{1} r_{2} \cdots r_{n-1}=0$; hence $x^{n}$ is a zero divisor in $R$, and so is $x$.

LEMMA 1.4 [3]. If $R$ is a periodic ring with $N \subseteq Z$, then $R$ is commutative.

2. The main theorems. We begin with results on rings having only finitely many noncentral subrings of zero divisors, which we will call $D$-nearly central rings (DNCrings). 
LEMMA 2.1. If $R$ is any $D N C$-ring, then $N \subseteq Z$ or $N$ is finite.

Proof. Assume $N \backslash Z \neq \phi$ and let $a \in N \backslash Z$. By [1, Lemma 3], $a$ has finite additive order and hence $a$ generates a finite noncentral subring of zero divisors. Thus, $N \backslash Z$ is finite; and since $u+N \cap Z \subseteq N \backslash Z$ for all $u \in N \backslash Z$, we see that $N \cap Z$ is also finite.

LEMmA 2.2. Let $R$ be an infinite DNC-ring with $N$ finite. Then every nil ideal of $R$ is central.

Proof. Suppose $I$ is a noncentral nil ideal. Since $I$ is finite, the two-sided annihilator $A(I)$ is of finite index in $R$, hence is infinite. Moreover, for any subring $S$ of $A(I), S+I$ is a noncentral subring of zero divisors of $R$; thus, there are only finitely many subrings $S+I$ with $S$ a subring of $A(I)$. It follows at once that $A(I)$ has only finitely many finite subrings, since otherwise there exist infinitely many distinct finite subrings $S_{1}, S_{2}, \ldots$, such that $S_{1}+I=S_{2}+I=\cdots=S_{n}+I=\cdots$ is a finite ring with infinitely many subrings.

It is well known that every infinite ring has infinitely many subrings (cf. [6]); hence $A(I)$ has infinitely many infinite subrings, and so does every infinite subring of $A(I)$. Thus $A(I)$ has an infinite strictly decreasing sequence $S_{1} \supset S_{2} \supset \cdots$ of subrings; and for some $j, S_{j}+I=S_{i}+I$ for all $i \geq j$. For each $i \geq j$, choose $a_{i} \in S_{i} \backslash S_{i+1}$ and write $a_{i}=b_{i+1}+c_{i}$, where $b_{i+1} \in S_{i+1}$ and $c_{i} \in I$. Since $I$ is finite, there exist $h, k$ such that $j \leq h<k$ and $c_{h}=c_{k}$. It follows that $a_{h}=b_{h+1}+a_{k}-b_{k+1}$ so that $a_{h} \in S_{h+1}$, a contradiction. Therefore, $I$ must be central.

LEMMA 2.3. If $R$ is any DNC-ring, then either $R$ is finite or $N \subseteq Z$.

Proof. Let $R$ be any infinite DNC-ring. By Lemmas 2.1 and 2.2, we may assume that $N$ is finite and $\wp(R) \subseteq Z$; and it follows by Lemma 1.3 that $\bar{R}=R / \wp(R)$ is a DNC-ring. By Lemma 1.2, $\bar{R}=B \oplus C$, where $B$ is an infinite reduced ring and $C$ is a direct sum of finitely many total matrix rings over finite fields. Suppose $C \neq\{0\}$ and contains the total matrix ring $K_{n}$ over the finite field $K$. We may assume $n>1$; otherwise $K_{n}$ can be incorporated into $B$. Now $B$ has infinitely many subrings; and for each subring $S$ of $B$, $S+K e_{1 n}$ is a noncentral subring of zero divisors of $\bar{R}$. Since $\bar{R}$ is a DNC-ring, we must therefore have $C=\{0\}$ so that $\bar{R}$ is reduced. It follows that in $R, N=\wp(R) \subseteq Z$.

REMARK 2.4. Theorem 1.1 follows at once from Lemmas 1.4 and 2.3. Thus, we have produced a proof of Theorem 1.1 which is very different from the one in [1].

We now arrive at our main result.

THEOREM 2.5. If $R$ is any ring with only finitely many noncentral subrings, then $R$ is finite or commutative.

Proof. Suppose $R$ is a counterexample with the minimum possible number of noncentral subrings. Then $R$ is infinite and every proper infinite subring of $R$ is commutative. Since $R$ is a DNC-ring, Lemmas 1.4 and 2.3 show that all finite subrings of $R$ are commutative as well; hence $R$ is a so-called one-step noncommutative ring-a noncommutative ring in which every proper subring is commutative.

Note that if $H$ is any set of pairwise noncommuting elements of $R$, the centralizers of the elements of $H$ are pairwise distinct noncentral subrings of $R$. Hence any set of 
pairwise noncommuting elements is finite, and by [2, Theorem 2.1], $Z$ has finite index in $R$. But by a theorem of Ikeda [4], a one-step noncommutative ring with $[R: Z]<\infty$ must be finite. Thus, we have contradicted our assumption that $R$ was a counterexample.

ACKNOWLEDGMENT. This work was supported by the Natural Sciences and Engineering Research Council of Canada, Grant 3961.

\section{REFERENCES}

[1] H. E. Bell and F. Guerriero, Some conditions for finiteness and commutativity of rings, Int. J. Math. Math. Sci. 13 (1990), no. 3, 535-544.

[2] H. E. Bell, A. A. Klein, and L. C. Kappe, An analogue for rings of a group problem of P. Erdós and B. H. Neumann, Acta Math. Hungar. 77 (1997), no. 1-2, 57-67.

[3] I. N. Herstein, A note on rings with central nilpotent elements, Proc. Amer. Math. Soc. 5 (1954), 620.

[4] M. Ikeda, Über die einstufig nichtkommutativen Ringe, Nagoya Math. J. 27 (1966), 371-379.

[5] A. A. Klein and H. E. Bell, Rings with finitely many nilpotent elements, Comm. Algebra 22 (1994), no. 1, 349-354.

[6] T. Szele, On a finiteness criterion for modules, Publ. Math. Debrecen 3 (1954), 253-256.

Abraham A. Klein: School of Mathematical Sciences, Sackler Faculty of Exact Sciences, Tel Aviv University, Tel Aviv 69978, Israel

E-mail address: aak1ein@post.tau.ac.i1

Howard E. Bell: Department of Mathematics, Brock University, St. Catharines, ON, Canada L2S 3A1

E-mail address: hbe11@brocku.ca 


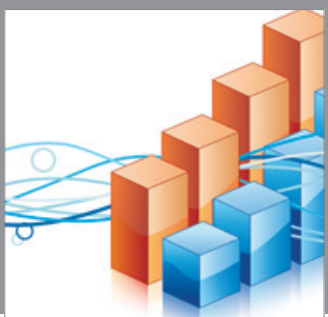

Advances in

Operations Research

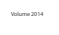

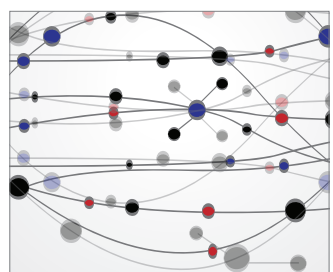

\section{The Scientific} World Journal
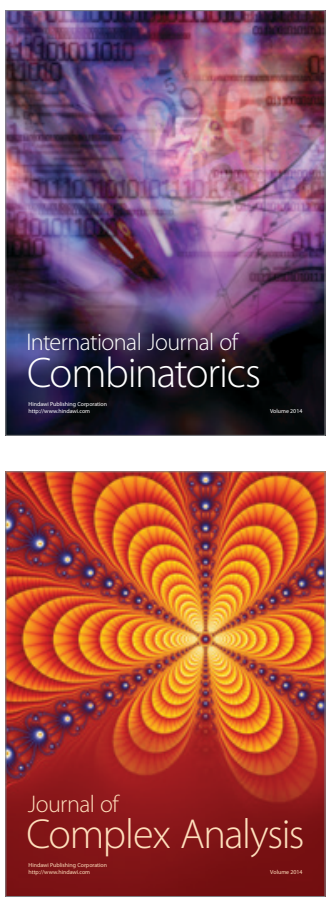

International Journal of

Mathematics and

Mathematical

Sciences
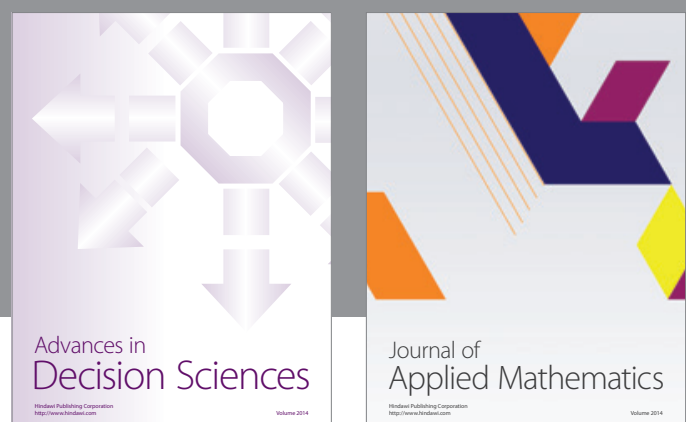

Journal of

Applied Mathematics
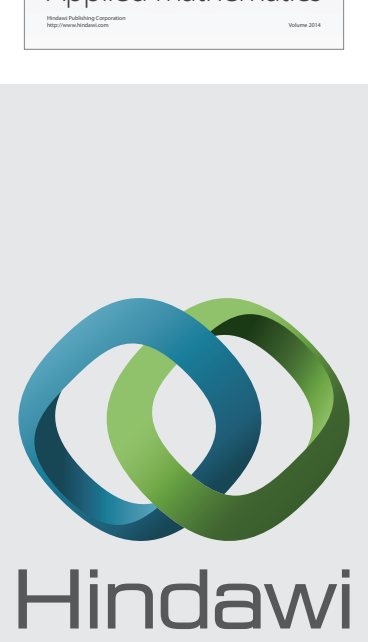

Submit your manuscripts at http://www.hindawi.com
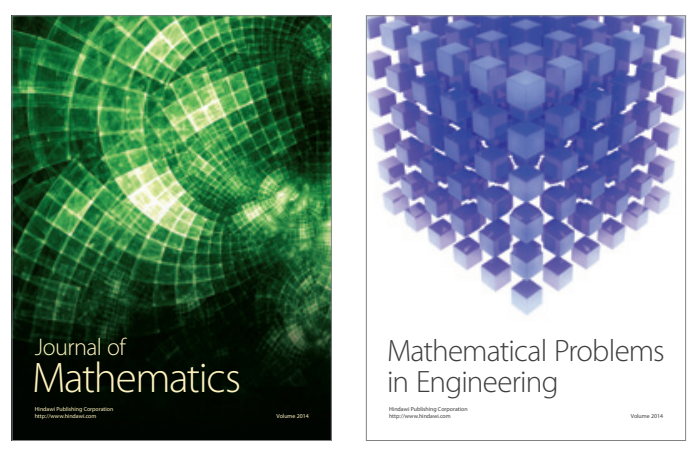

Mathematical Problems in Engineering
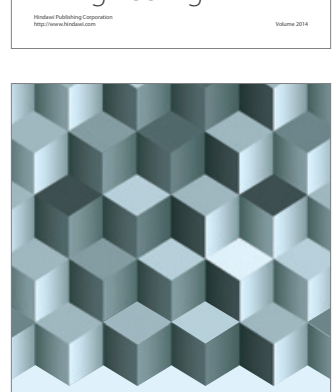

Journal of

Function Spaces
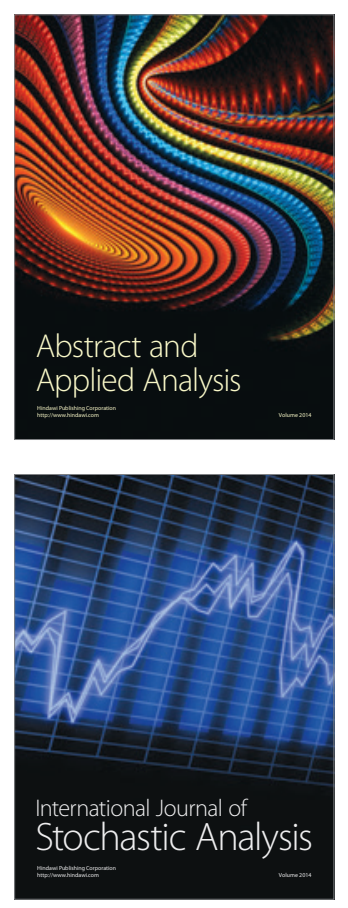

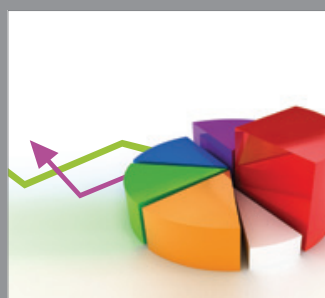

ournal of

Probability and Statistics

Promensencen
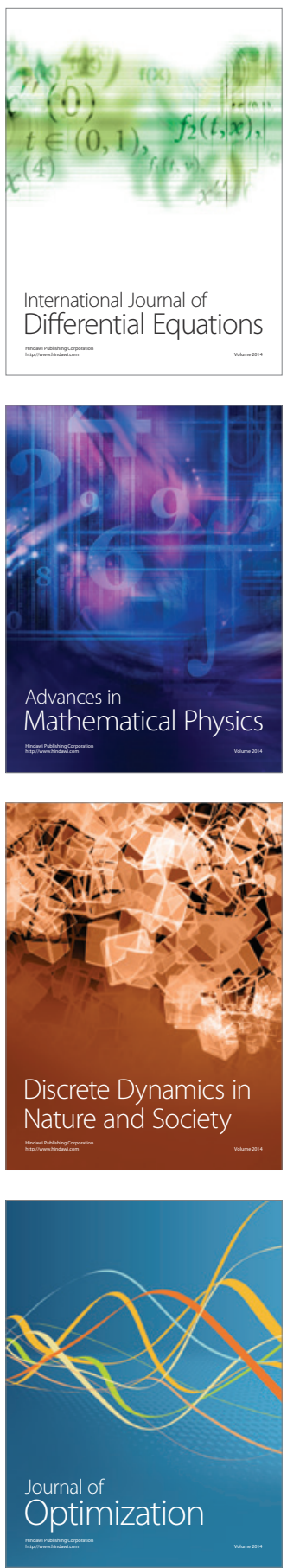